\title{
Optimization of Solid Oxide Fuel Cell Cathodes Using Two-Point Correlation Functions
}

\author{
Ali Hasanabadi ${ }^{1}$, Majid Baniassadi ${ }^{1 *}$, Karen Abrinia ${ }^{1}$, Masoud Safdari ${ }^{2}$ Hamid Garmestani $^{3}$ \\ ${ }^{1}$ School of Mechanical Engineering, College of Engineering, University of Tehran, P.O. Box 111554563, Tehran, Iran \\ ${ }^{2}$ Aerospace Engineering Department, University of Illinois, 104 S Wright St., Urbana, IL 61801, USA \\ ${ }^{3}$ School of Materials Science and Engineering, Georgia Institute of Technology, USA
}

\begin{abstract}
A novel method for the optimization of the microstructure of a two-phase solid oxide fuel cell (SOFC) mixed ionic-electronic conductor (MIEC) cathode is presented. Two-point correlation functions (TPCFs) are used to manipulate the microstructure. At first, using an appropriate function, such as a decaying exponential multiplied by a sinusoidal function, initial full-set TPCFs are created. Based on the created TPCFs, a phase recovery algorithm is used, as a construction tool, to realize the three-dimensional (3D) porous microstructure of the SOFC. The reconstructed microstructures are evaluated based on the contribution of the geometrical attributes, such as tortuosity of the solid-phase and the active interfacial area, minimizing the cathode characteristic impedance. Shooting for a minimum solid-phase tortuosity and maximum active interfacial area of solid- and void-phase for a fixed volume fraction, a series of optimization simulations are carried out using two independent variables of autocovariance function as the design variables. Using this approach, it is possible to create very thick (or thin) pathways in the solid and void phases with small (or large) tortuosity and low (or high) interfacial area of the solid-void by applying various TPCF sets. By comparing the present results with some of the experimental ones reported in the literature, it is shown that the optimization process presented here can be used as a robust tool to design optimal microstructure with improved tortuosity and interfacial area for SOFCs and other similar bicontinuous applications.
\end{abstract}

Keywords: two-point correlation functions; optimization of the microstructure; tortuosity; active interfacial area; solid oxide fuel cell; phase recovery algorithm; mixed ionic-electronic conductor cathode

\section{Introduction}

Solid oxide fuel cells (SOFCs) are energy conversion devices, converting chemical energy of fuels (such as hydrogen, hydrocarbons, carbon monoxide etc.) to electrical energy with high efficiency compared with traditional technologies. These energy converters do not necessarily use Carnot cycle, thus they can reduce the emission of pollutants and overcome combustion efficiency limitations [1]. Because of these compelling advantages, many research works are carried aiming different aspects of these devices such as microstructure optimization [2-5] and reconstruction [6-9].

Solid oxide fuel cell consists of three parts: a cathode, an anode, and an electrolyte, each with a specified task in the process of converting chemical energy into electricity. In the cathode, oxygen molecules diffuse through the void

*Corresponding author, m.baniassadi@ut.ac.ir

(C) 2016. This manuscript version is made available under the Elsevier user license http://www.elsevier.com/open-access/userlicense/1.0/ 
pores, get converted to ions and transferred to the anode by a solid ion-conducting electrolyte. After crossing the electrolyte, oxygen ions react with hydrogen molecules in the anode. The result of this reaction is water associated with two free electrons that flow through the circuit.

The role of SOFC cathode is to provide a site for the reduction of the oxygen. So it must have appropriate properties such as high electronic conductivity, adequate porosity to allow the diffusion of oxygen into the cathode-electrolyte interface, high catalytic activity, chemical and thermal compatibility with electrolyte, stability in high temperature, and it should be low cost [10]. Among these, current study is focused on the improvement of the diffusion of oxygen ion through the solid-phase and increasing the interfacial area of the solid and void phases.

Generally, SOFC cathodes should have maximum triple-phase boundary, where the electron conductor (cathode), the ion conductor (electrolyte) and the gas (void) meet each other. The cathode microstructure is usually made from one of the three compositional designs: (i) an electronic conductor such as LSM (LaSrMnO3); (ii) a composite of LSM and YSZ (yttria-stabilized zirconia); and (iii) a mixed ionic-electronic conductor (MIEC) [10]. MIEC cathode electrode is a single-phase electrode with mixed conductivities that provides both electron mobility and oxide ion conductivity within the cathode material. Example of this electrode is LSCF ( $\mathrm{LaSrCoFeO} 3$ ) [11].

The generation and transport of oxygen ions in the cathode (porous media) is associated with resistance losses. Two approaches are suggested to reduce such losses [10, 12]: (i) development of electrode with high ionic conductivity using a novel material, bearing in mind that the thermal and chemical properties of the cathode should be matched with other components [13,14]; and (ii) rational design and optimization of the cathode microstructure in order to extend the TPB length and increase effective diffusion coefficient of the oxygen gas. The latter approach is adopted in the current study.

In LSM and composite LSM-YSZ cathodes, triple-phase boundary is a line and the presence of LSM, YSZ and void is important. But for MIEC cathodes the surface area of sloid phase is important [15, 16], mainly because of the mixed ionic and electronic conductivity.

Endo et al. [16] measured electrochemical characteristics of the LSM and LSC (LaSrCo3) electrodes, and they found that the surface area is an important factor for the reaction rate on the LSC electrodes. Their study results suggested that high performance electrodes could be obtained with a high ionic conductor electrode with a large surface area. For the MIEC cathodes, a high surface area provides more active sites for the oxygen reduction leading to an improved performance [10]. Song et al. [3] proposed a 2D model for the shape optimization for the solid oxide fuel cell cathodes. They used a simplified model and topology optimization to reduce the ohmic losses and minimize resistance by optimizing the shape of the cathode. Farhad et al. [4] developed a micromodel to predict the electrochemical performance of porous composite LSM-YSZ cathodes in solid oxide fuel cells. In their study, factors such as mass transport, electron and ion transport, and the electrochemical reaction were used as the criteria for the optimization of the microstructure of the porous LSM-YSZ composite cathodes. They concluded that the electrochemical performance of the cathode depends on the microstructural variables such as porosity, thickness, particle size ratios, and size and volume fraction of the LSM particles.

The manipulation of a microstructure based on its two-point correlation functions (TPCFs) can be used to obtain a wide variety of microstructures. Jiao et al. [17] used this approach to construct microstructures with desired 
structural characteristics. Similarly, Bodla et al. [18] utilized a parametrized model for the design of porous materials based on two-point autocorrelation functions to reconstruct sample 3D microstructures, and they analyzed the influence of various parameters on fluid-thermal properties of interest.

In the current work, a new procedure is presented for optimization of microstructure of SOFC cathodes using statistical approaches such as n-point correlation functions. Due to the inherent capability of the n-point correlation functions in controlling the geometry and properties simultaneously, they are applied to construct different cathode microstructures with diverse geometrical properties and therefore a suitable search space is provided. The construction process consists of two stages: (i) based on two independent variables, two-point correlation functions (TPCFs) of the microstructure are calculated; and (ii) a 3D and two-phase porous media is realized based on TPCFs using phase recovery algorithm. The evaluation of the microstructures is carried out based on maximizing the active interfacial area and tortuosity of solid-phase subject to a constraint on the maximum void-phase tortuosity. It is noteworthy that the proposed procedure for microstructural optimization fundamentally differs from the conventional approaches in structural optimization particularly in the provided search space. In more conventional methods, such as topology optimization [19-22], all voxels of the discretized microstructure are considered as design variables so taking small base cell is inevitable, however, in the proposed optimization procedure, TPCFs space, parametrized by two variables, is utilized as the search space which considerably accelerate the optimization process and it is possible to consider relatively large design domain. It should be noted that the proposed procedure is applicable for a wide range of bicontinuous microstructure where percolation and conductivity of the both phases is desired simultaneously. Simplicity and elegance are two main features of the proposed approach which make it a robust method to find the relationship among the TPCFs, the microstructure and its properties.

\section{Describing the Microstructure Using Statistical Correlation Functions}

\subsection{N-point Correlation Functions Definition}

First stage in the manipulation of the geometrical attributes of a microstructure is to describe it properly in a parametric design space. Statistical descriptors such as correlation functions are appropriate tools for this purpose when a microstructure is statistically homogeneous. N-point correlation function is an appropriate statistical descriptor for characterizing microstructure morphology [23, 24], and it is shown that the effective properties of random heterogeneous materials such as mechanical, thermal, electrical and permeability can be strongly correlated with the n-point statistics [23-26].

$\mathrm{N}$-point correlation function is the probability of finding an arrangement of $\mathrm{N}$ points in a specific phase (or in an arrangement of phases). The simplest form of n-point correlation functions is one-point function that is the probability of finding a randomly chosen point in a specified phase. In the asymptotic limit, the one-point correlation of each phase is equivalent to its volume fraction, and it conveys no information about microstructure geometry. With increasing the order of correlation functions, the extent of geometric information captured by the function is 
increased, such that every microstructure can be uniquely reconstructed from its full-set infinite-order correlation functions [27-29].

Two-point correlation function is the probability of finding the head and tail of a vector connecting two randomly chosen points in the specified phases. These functions describe a joint probability, and are the simplest form of the correlation functions that capture a basic level of the geometrical attributes of a microstructure. For a statistically homogeneous microstructure, two-point functions are invariant with respect to the absolute position of the points. Therefore for such microstructure a point is chosen as origin and other point is found by throwing a random vector originating from this point.

For a randomly thrown vector, $\vec{r}$, with a head and a tail, denoted by $x_{1}$ and $x_{2}$ respectively, the TPCF $C_{2}^{i j}(\vec{r})$ can be expressed as:

$$
C_{2}^{i j}\left(x_{1}, x_{2}\right)=C_{2}^{i j}(\vec{r})=P\left\{\left(x_{1} \in i\right) \cap\left(x_{2} \in j\right)\right\}, i, j \in \text { set of phases. }
$$

For eigen microstructures, every point is only occupied by one phase and can be represented by the characteristic function as:

$$
p_{x}^{i}=\left\{\begin{array}{rr}
1 & x \text { in phase } i \\
0 & \text { otherwise }
\end{array}\right.
$$

Considering $\mathrm{N}$ points within the microstructure, randomly positioned at $x_{1}, x_{2}, \ldots, x_{N}$, the probability of finding them in the phases $i, j, \ldots, m$ is defined by the n-point correlation function (see Fig. 1):

$$
C_{N}^{i j \ldots m}\left(x_{1}, x_{2}, \ldots, x_{N}\right)=\left\langle p_{x_{1}}^{i} p_{x_{2}}^{j} \ldots p_{x_{N}}^{m}\right\rangle, \quad i, j, \ldots, m \in \text { set of } p h a s e s
$$

where $\langle\ldots\rangle$ symbol represents an ensemble average.

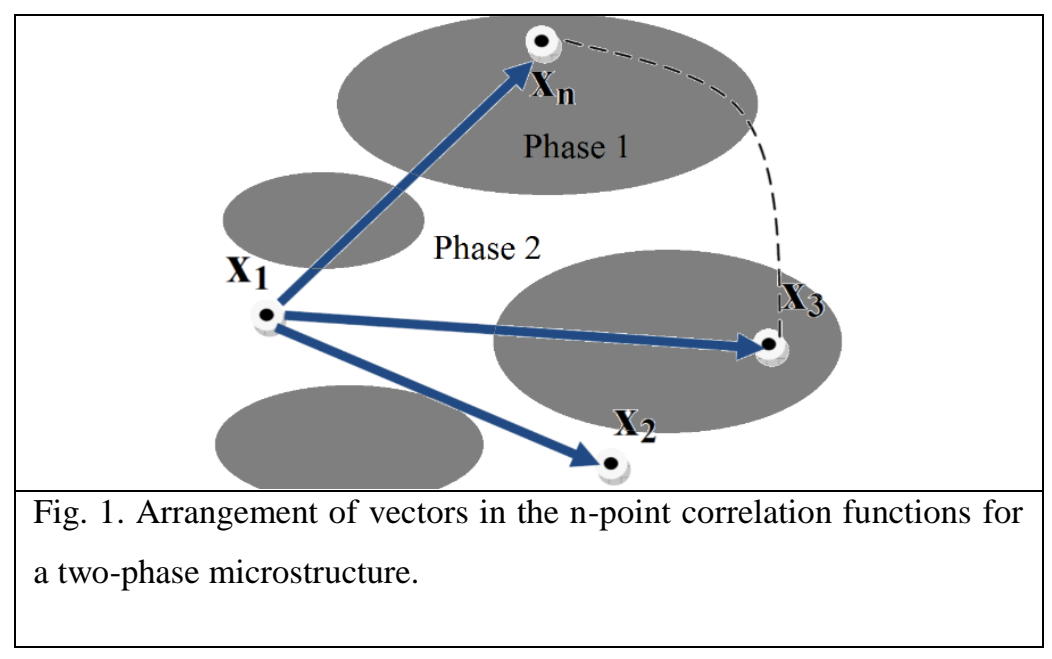


For a two-phase microstructure, $C_{2}^{11}, C_{2}^{22}, C_{2}^{12}$, and $C_{2}^{21}$ are four interdependent TPCFs. For a two-phase media, there is only one independent set of TPCFs, and all other sets can be expressed in terms of that one.

Due to the conditions of TPCFs, the following constraints can be satisfied for a n-phase microstructure [30]:

$$
\begin{gathered}
\sum_{i=1}^{n} \sum_{j=1}^{n} C_{2}^{i j}\left(x_{1}^{i}, x_{2}^{j}\right)=1 \quad \text { where } \vec{r}=x_{2}^{j}-x_{1}^{i} \\
\sum_{j=1}^{n} C_{2}^{i j}\left(x_{1}^{i}, x_{2}^{j}\right)=v_{i} \\
C_{2}^{i j}\left(x_{1}^{i}, x_{2}^{j}\right)=C_{2}^{j i}\left(x_{2}^{j}, x_{1}^{i}\right)
\end{gathered}
$$

where $v_{i}$ is volume fraction of the phase $i$.

\subsection{Calculation of TPCFs for Cathode Microstructure}

An optimization procedure to find an optimal microstructure requires a tool to construct the microstructures. Here, the construction procedure is carried out based on the hypothetical TPCFs of the desired microstructure. An arbitrary function is not necessarily a correlation function for a physically realizable microstructure. A valid correlation function must satisfy certain conditions as described in section 2.1. In addition to general constraints, Eqs. (4)-(6), a realizable set of desired TPCFs must meet non-negativity and inequality conditions. Torquato et al. [23, 31, 32] investigated these conditions for a certain class of functions known as autocovariance functions. Autocovariance function of vector $\vec{r}$ is defined based on its TPCF by:

$$
f_{2}^{i}(\vec{r})=\frac{C_{2}^{i i}(\vec{r})-v_{i}^{2}}{v_{i}\left(1-v_{i}\right)}
$$

One simple function that satisfies non-negativity and inequality constraints [32] is the exponentially decaying function that can expressed as:

$$
f(r)=\exp \left(-\frac{r}{b}\right), \quad r=|\vec{r}|
$$

where $b$ is a positive characteristic parameter. For larger $b$ values, the size of the microstructure phases will be coarser (see Fig. 5). It should be noted that the constructed microstructures here are assumed to be isotropic in all directions. So only the length of vector $\vec{r}$ is considered in Eqs. (8) and (9). This function was first proposed by Debye et al. [33] for structures in which a phase possesses random shape and size. This monotonically decreasing function neglects short-range orders, in other words it does not exhibits any oscillations for sufficiently large $r$ values [23]. 
Another function that is a generalization of the Debye function and also exhibits short-range order (i.e. exhibits oscillations for small $r$ ) can be expressed by multiplying Debye function with an oscillating sine function:

$$
f(r)=\exp \left(-\frac{r}{b}\right) \frac{\sin (q r)}{q r}
$$

where $q$ is a parameter that controls the oscillations in the function. Note that the function is parametrized by two variables to represent a more diverse range of microstructures. This function is first proposed by Cule and Torquato [34] for constructing amorphous material, such as porous media, using hypothetical TPCFs [34]. It also exhibits phase inversion symmetry [23]. A two-phase random medium possesses phase-inversion symmetry if the morphology of phase 1 at volume fraction $v$ is statistically identical to that of phase 2 where the volume fraction of phase 1 is $1-v$ [23]. This property improves the bicontinuity or simultaneous percolation of both phases of a twophase microstructure [23].

This autocovariance function satisfies non-negativity and inequality conditions (except for one inequality) for volume fraction interval [32]:

$$
0.1538<v_{i}<0.8461
$$

In the current study, this function is selected as the underlying parametric TPCFs for the manipulation and optimization of the microstructure because it represents an appropriate bicontinuous porous media [23, 34, 35], realizes a long range of volume fractions, and satisfies almost all necessary conditions. It is noteworthy that the evaluation of sufficient conditions for the realizability of autocovariance functions is a subtle problem. As the realization of an optimum microstructure is the main objective of this study, the microstructure is constructed solely based on this assumption and, therefore, in a subsequent step, the reconstructed microstructure is checked to make sure it satisfies the desired volume fraction. The constructed microstructure will be accepted if its volume fraction satisfies the desired value.

\section{Construction Procedure}

Realization of microstructure based on its statistical correlation functions is carried out using various numerical methods such as simulated annealing [17, 23, 35], Gaussian random fields [36], gradient-based schemes [37] and phase recovery method $[38,39]$. Among these diverse methods, the phase recovery matches well with TPCFs, and converges rapidly to the desired microstructure with more efficiency.

Phase recovery method, originally proposed by Gerchberg and Saxton [40], is best applicable to the problems with known modulus and unknown phase information such as X-ray crystallography, image processing and microstructure construction. The algorithm employed here is based on the method proposed by Fienup [41] that can be used for the general purpose of reconstruction from the modulus of a Fourier transform. 
For a digitized media, the construction procedure based on phase recovery algorithm, utilizes the discrete Fourier transform [38] and the characteristic function (Eq. (2)) to construct the microstructure. For every point $x$, the characteristic function, $p_{x}^{i}$ is the probability of the occupation of the position $x$ with phase $i$ and is zero or one for an eigen microstructure. So for a prescribed position $x$, it can be stated that:

$$
\sum_{i=1}^{n} p_{x}^{i}=1, \quad p_{x}^{i} \in\{0,1\}
$$

where $n$ denotes the number of phases in the microstructure.

By using characteristic function, one-point correlation functions for a specified phase $i$ can be expressed as:

$$
C_{1}^{i}=\frac{1}{Z} \sum_{x=0}^{Z-1} p_{x}^{i}
$$

where $Z$ is the total number of grid points of the microstructure. Similarly, two-point correlation function for a specified vector, $r$, can be expressed as:

$$
C_{2}^{i j}(r)=\frac{1}{Z} \sum_{x=0}^{Z-1} p_{x}^{i} p_{x+r}^{j}
$$

where the superscripts $i$ and $j$ denote the phases of interest.

Phase recovery algorithm uses Fourier transform in the construction procedure [38]. By applying fast Fourier transform (FFT) on Eq. (12) for all point $x$ and fixed phase $i, P_{m}^{i}$ can be expressed as:

$$
P_{m}^{i}=\mathcal{F}\left(p_{x}^{i}\right)=\frac{1}{Z} \sum_{x=0}^{Z-1} p_{x}^{i} \mathrm{e}^{2 \pi k x m / Z}=\frac{1}{Z}\left|P_{m}^{i}\right| \mathrm{e}^{k \theta_{m}^{i}}, \quad k=\sqrt{-1}
$$

where $\left|P_{m}^{i}\right|$ and $\theta_{m}^{i}$ are the amplitude and phase of the Fourier transform, respectively. Assuming periodicity of the structure and applying convolution theorem, fast Fourier transform of two-point correlation function can be obtained by:

$$
P_{m}^{i j}=\mathcal{F}\left(C_{2}^{i j}(r)\right)=\frac{1}{Z}\left|P_{m}^{i}\right| \mathrm{e}^{-k \theta_{m}^{i}}\left|P_{m}^{j}\right| \mathrm{e}^{k \theta_{m}^{j}}
$$

In Eq. (15) if the head and tail of a vector $r$ reside in phase $i$ only the amplitudes of FFT remain which can be expressed as:

$$
P_{m}^{i i}=\frac{1}{Z}\left|P_{m}^{i}\right|^{2}
$$


Using this equation, it is possible to express FFT of the two-point correlation function of phase $i$ in terms of the modulus of the FFT of the characteristic functions of the microstructure. Given the known TPCFs of the microstructure for the specified phase $i$, the modulus of entire points in FFT space will be determined. Hence it is sufficient to recover the phase of $P_{m}^{i}$ or $\theta_{m}^{i}$, to determine the entire microstructure functions for all points and the specified phase $i$.

The algorithm that is used here for reconstruction, following Fineup phase recovery algorithm [41], has four essential steps: (i) an initial guess for the microstructure is used to initialize the algorithm and calculate the FFT using Eq. (14), (ii) the modulus of $P_{m}^{i}$ is replaced with the $\left|P_{m}^{i}\right|$, that is calculated using Eq. (16) and the phase is kept unchanged, (iii) the inverse FFT of the corrected $P_{m}^{i}$ is calculated based on Eq. (14), (iv) any constraint in the real space, such as Eq. (2), is imposed in the last step. The output microstructure of this step is used as input microstructure for step 1. After each iteration, the TPCFs of the microstructure can be determined using Eq. (13) and the error between reference TPCFs and constructed ones can be calculated. The algorithm stops when a criteria (some measure of error) is satisfied.

\section{Optimization Process}

\subsection{Search Space}

Optimization process consists of three main stages: (i) initial assumption about the full set of TPCFs; (ii) construction of the microstructure based on assumed TPCFs using phase recovery algorithm; and (iii) evaluation of the constructed microstructure. Supposed TPCFs are the initial step of the optimization procedure, and this space is generated using Eqs. (7) and (9). Using two variables $b$ and $q$, it is possible to obtain various microstructures. An important question arises here, for what values of $b$ and $q$, it is possible to calculate a feasible set of TPCFs? Torquato outlined the necessary conditions such as non-negativity and inequalities conditions for the autocovariance function of Eq. (9) and expressed that this function satisfies all known necessary condition, but triangular inequality that causes this function is not exactly realizable [32]. For the purposes of the current study an approximate, but systematic, realization of autocovariance is sufficient. The only constraint that the constructed microstructure must satisfy is the specified volume fraction. So any pair of $(b, q)$ for which the constructed microstructure does not violate the volume fraction constraint, can be acceptable. 


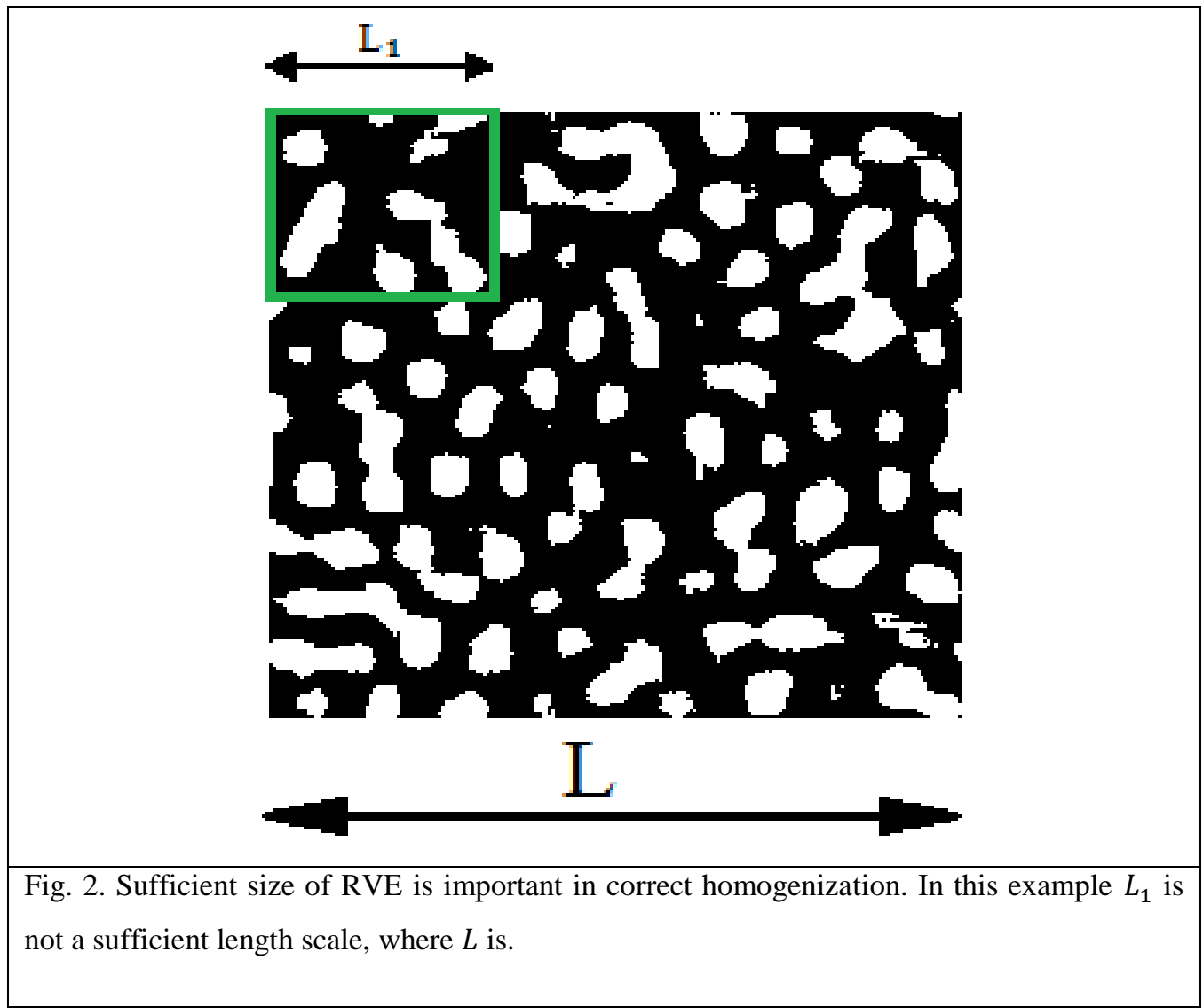

Two important factors that should be set up in the initial step of the procedure, are the representative volume element (RVE) size and the number of its voxels. Indeed, RVE is the microstructure design space. If the constructed RVE is not large enough (compared with the size of each of the phases), the targeted properties, obtained by homogenization method, are not reliable. For example green square (with length $L_{1}$ ) in Fig. 2 is not appropriate as $\mathrm{RVE}$ of the microstructure, but that with length $L$, properly represents the property of the microstructure.

Number of voxels is also important because although $r$ in autocovariance function, varies between zero and the RVE size ( $L$ in Fig.2), a poor voxelized representation neglects the details of the autocovariance function.

In the current study, for a specific volume fraction of void phase, $v=0.41$ and a RVE size of 200 voxels in each direction, by choosing a specified length such as $L=7.5$, the details of the autocovariance functions are considered properly, and the resulted microstructures (for some random pairs of $(b, q)$ where $b, q>0$ ) are satisfactory.

For the objective function established in section 4.2, the tortuosity of void-phase will be considered as a constraint. It is shown in the next sections that for $q>40$ and $b>51.2$, this constraint is undoubtedly violated. So the intervals of $0<q \leq 40$ and $0<b \leq 51.2$ constitute the search space here. In order to reduce the calculation time, 70 pairs of $(b, q)$ are selected in these intervals, and for these points the autocovariance functions and TPCFs are calculated using Eqs. (7) and (9). In the following, the RVEs are constructed based on the calculated TPCFs and an 
objective function (details will be described in the next section) is evaluated for every RVE. Using a neural network for other points of the search space the objective function is approximated. By searching this space for the maximum value of objective function and considering its constraint, the optimal point is found. After finding the optimal pair $(b, q)_{\text {opt }}$, the optimal microstructure is reconstructed.

Construction of the RVEs, for the specified intervals reveals that there is a high diversity in the size and quality of the geometrical attributes (see Fig. 3). The diversity in the constructed RVEs causes a wide range of effective properties and, therefore, a suitable search space is provided for the optimization of objective function.

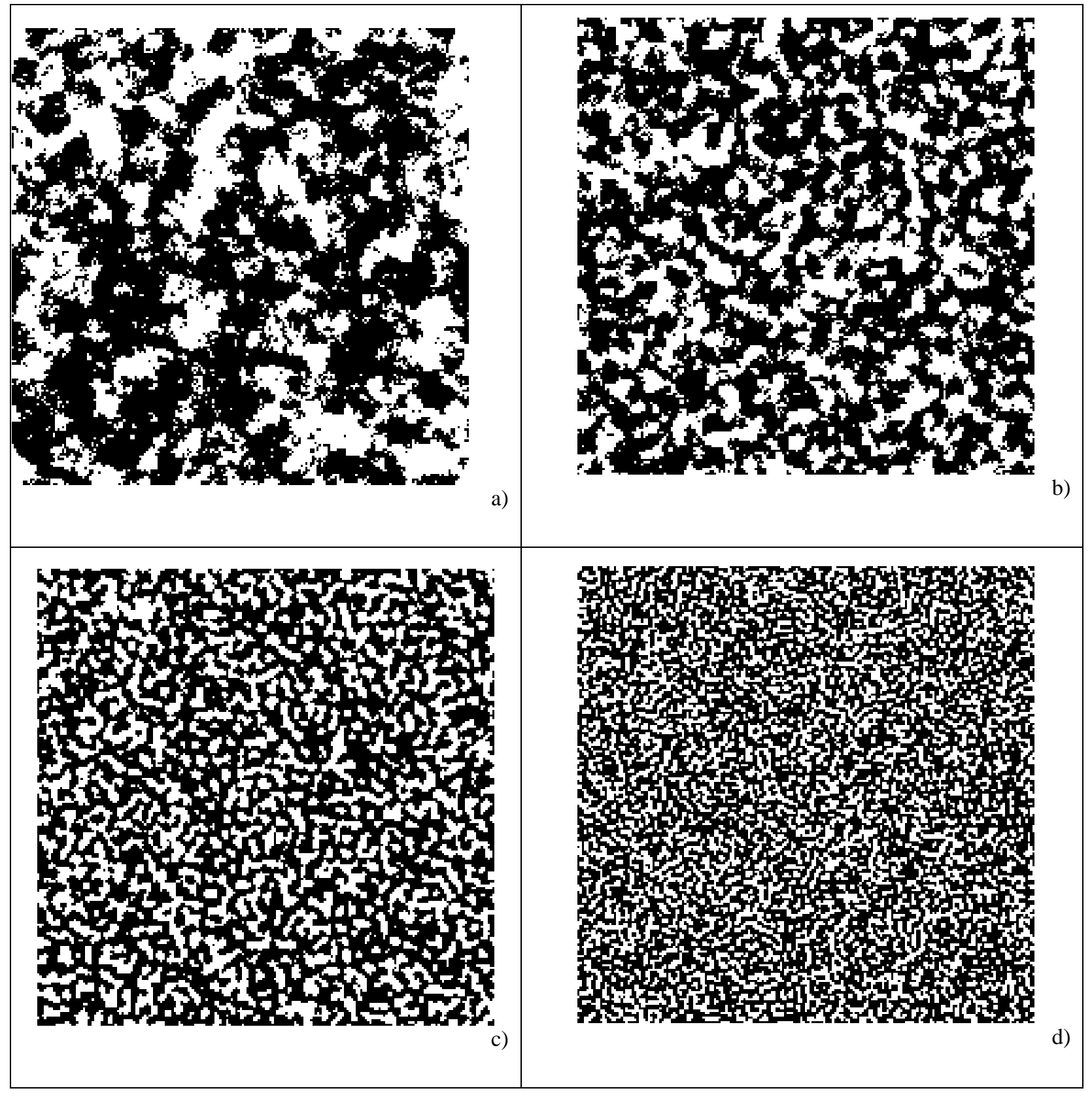


Fig. 3. Diverse microstructures that are constructed for $v=0.41, b=0.4$ and $q=2.5$ (a), 7.5 (b), 15 (c), 25 (d).

\subsection{Objective Function Establishment}

In this work, the microstructural configuration of the mixed ionic-electronic conductor cathodes (such as LSCF) is investigated. In the cathode two reactions are usually considered: (i) the reduction of oxygen molecules and (ii) ionic transport of the anions through the solid-phase toward the electrolyte. These reactions are the biggest contributors to the total cell resistance $[10,11]$ and any improvements on the catalytic activity of the cathode (such as microstructure optimization) have a profound impact on the final cell performance.

Adler et al. [11] used continuum modeling for MIEC cathodes with fast ionic transport (such as LSCF) and indicated that solid-state oxygen diffusion and $O_{2}$ surface exchange, dominate the electrochemical behavior, and produce effective chemical resistance. For a thick electrode, with no gas-phase limitations, the characteristic resistance or the total impedance of the steady state is described by [11]:

$$
R_{\text {chem }}=\frac{R T}{2 F^{2}} \sqrt{\frac{\tau}{(1-v) a C_{0}^{2} D k}}
$$

where $\tau, v$ and $a$ are tortuosity of solid phase, volume fraction of the void phase and interfacial surface area for a unit volume, respectively. Also $C_{0}, D$ and $k$ are surface concentration of the oxygen, oxygen self-diffusion coefficient, and oxygen surface exchange coefficient, respectively. $R$ and $F$ are constants and $T$ is temperature.

Eq. (17) is reliable for $P_{O_{2}}>0.1 \mathrm{~atm}$. For oxygen pressure below this value, gas-phase diffusion resistance can become significant and should be considered [11].

Therefore for a MIEC electrode with facile mass transport, the contribution of geometrical arrangement of the microstructure for a specified volume fraction and material (with prescribed $C_{0}, D$ and $v$ ) can be optimized by optimal values for $\tau$ and $a$.

Tortuosity of solid or void phase of the porous media can be calculated using a reasonable approximation for the effective diffusion coefficient described by [12, 42]:

$$
\tau=(1-v) \frac{D}{D^{e f f}}
$$

where $D^{e f f}, \tau$ and $v$, are effective diffusion coefficient, tortuosity of solid phase, and volume fraction of void phase, respectively. For a fixed volume fraction of the void phase, the tortuous channels increase notably the diffusion length and the resistance of ion transport.

Effective diffusion coefficient of a distinct phase is mathematically considered equivalent to the effective electrical and thermal conductivity problems when other adjacent phases are insulator to what passes through the specified 
phase [23]. In the case of SOFC cathode, oxygen anions only transport through the solid phase. Therefore, it is possible to use this analogy for calculating tortuosity using effective thermal conductivity coefficient (assuming that heat flux only transport through solid phase).

Using mathematical equivalence of thermal and diffusion problems, a dimensionless factor can be defined by:

$$
F=\frac{\sigma}{\sigma^{e f f}}=\frac{D}{D^{e f f}}
$$

where $\sigma^{e f f}$ and $\sigma$ are the effective and bulk thermal conductivity coefficients of the RVE, respectively. By solving thermal conductivity problem in three directions and assuming $\sigma=1, \sigma^{e f f}$ and $F$ factor can be calculated for each direction. For any specified direction two surfaces, perpendicular to the considered direction, are assigned different temperatures as boundary conditions and all other surfaces are assumed to be insulated. An average $F$ factor, based on three directions, is considered as $F$ factor for every RVE.

Using Eqs. (18) and (19) tortuosity can be expressed as:

$$
\tau=F(1-v)
$$

It is worth mentioning that for any specified volume fraction, $F$ factor (or tortuosity) is a geometrical characteristic of the microstructure, and it does not depend to the boundary conditions or physical properties of the bulk material. Another important factor is the interfacial area of the void- and solid-phase. By increasing the interfacial area, the oxygen reduction sites can be increased. Therefore based on Eq. (17), the objective function that is used to find the optimal design of the cathode microstructure for a fixed volume fraction and minimum impedance, can be expressed as:

$$
\text { of }=\tau^{-1} a_{\text {active }}
$$

where $a_{\text {active }}$ is the interfacial area of the active clusters of the void- and solid-phase. A cluster of a phase is defined as the subset of that phase for which every selected two points, can be joined to each other without crossing other phases. If a cluster connects two opposite surfaces of the RVE, this cluster is considered as an active cluster in the direction perpendicular to the mentioned surfaces.

Using an algorithm based on cluster multiple labeling technique [43], every voxel occupied by the solid- or voidphase is checked whether it belongs to an active cluster or not. Then, in order to determine the active interfacial area, using another algorithm, the number of interfacial faces between void- and solid-phase is counted for all voxels. To avoid recounting a face, on each plane that is searched, only the right and top faces of the voxels are examined (Fig. 4). For each of the three directions and for all planes perpendicular to each direction, counting operation, is performed. 


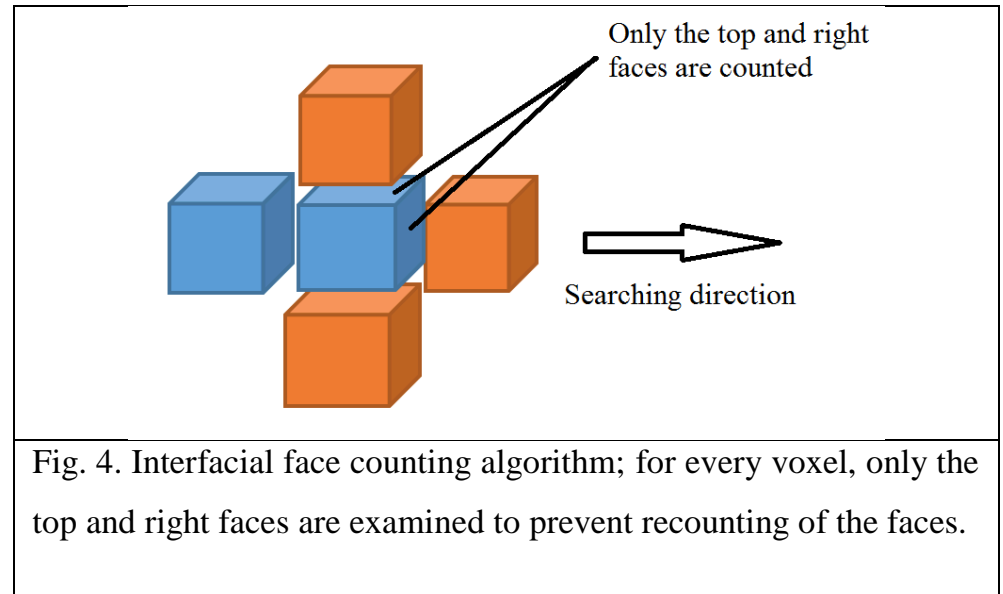

It should be noted that facile gas transport is an essential issue in deriving Eq. (17) [11]. The void-phase of the porous media provides pathways for flowing oxygen gas through the structure. The mechanism of gas transport in the electrodes of SOFC is mainly controlled by diffusion because its porous structure has the pore size of nanometer-to-micrometer scale [12]. Therefore in order to satisfy this condition, in the process of optimization of the microstructure, based on the objective function of Eq. (20), the tortuosity of void-phase should not exceed a maximum quantity.

Various values for tortuosity of void-phase have been reported in the literature. Based on an experimental study on the tortuosity of the MIEC cathode with the same material, Cooper et al. [42] reported the maximum value for tortuosity of void-phase to be 3.45. Therefore the optimization problem is expressed as maximizing the Eq. (20) with the constraint that the value of void-phase tortuosity does not exceed 3.45.

\section{Results}

\subsection{Debye Autocovariance Function}

For the single parameter Debye function, Fig. 5 demonstrates the tortuosity of solid- and void-phase and the active interfacial faces. It can be seen that by increasing the characteristic parameter $b$, the tortuosity of the solid-phase and the active interfacial faces decrease to asymptotic values and the tortuosity of the void-phase decreases to a minimum value, and then begins to increase. Also the sizes of solid and void pathways become thicker for larger values of $b$. 


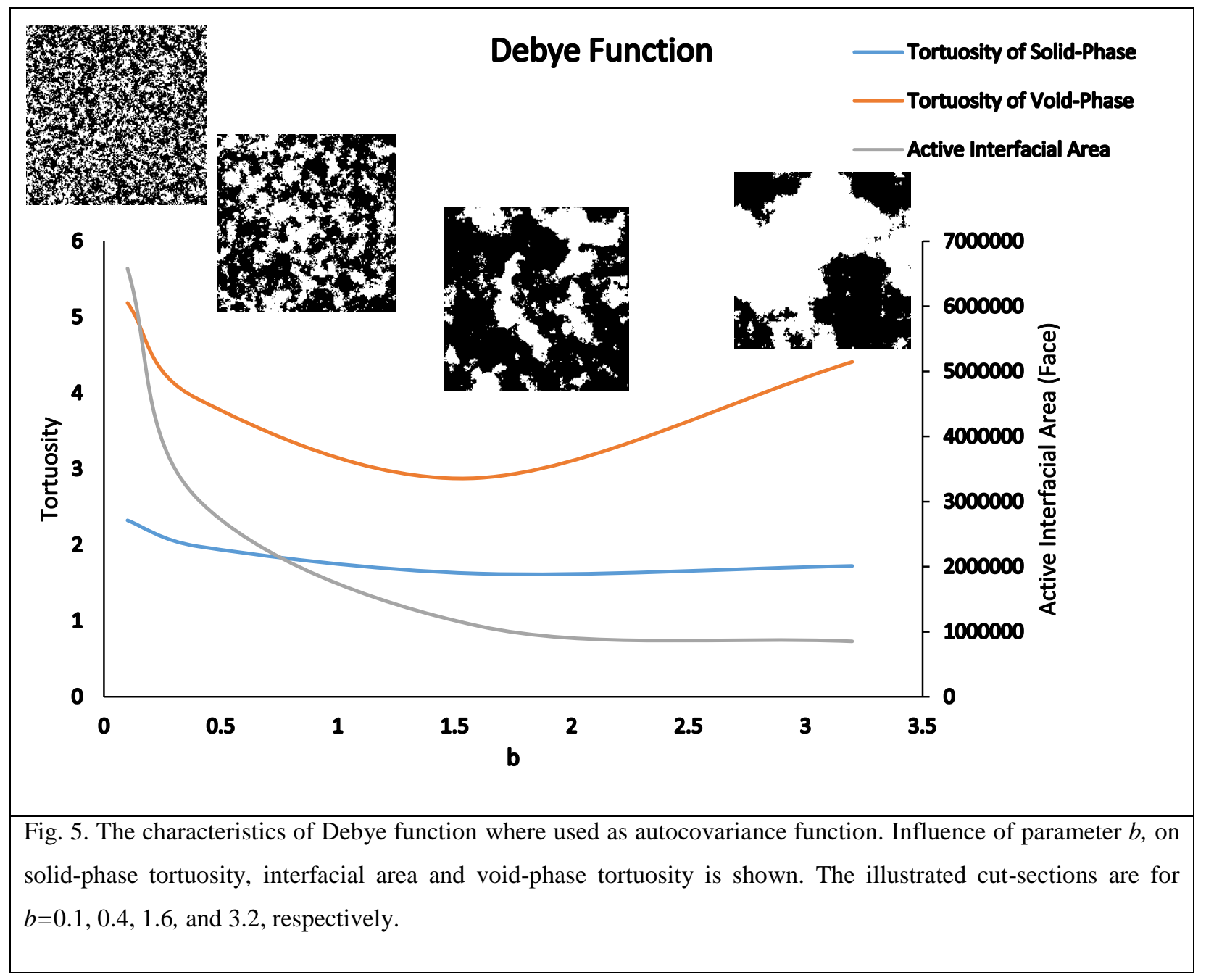

\subsection{Damped-Oscillating Autocovariance Function}

By searching the space described in section 4.1, and evaluating the objective function (Eq. (20)), it is revealed that by increasing $q$ factor in Eq. (9) for all $b$, the dimensions of solid- and void-phase pathways become finer. This refinement causes the interfacial area to increase, and thus the efficiency of the cathode is improved (Fig. 6). On the other hand, refinement of microstructure increases the tortuosity and gas diffusion resistance through the void-phase (Fig. 6). Compared with void-phase, tortuosity of solid-phase changes to a less degree. This is because of the larger volume fraction of the solid-phase (0.59 compared to 0.41) that causes geometrical arrangement of the microstructure to affect the tortuosity of the solid-phase to a lesser extent. Also Fig. 7 illustrates the microstructure variations and the objective function for $b=1.6$ versus $q$. 

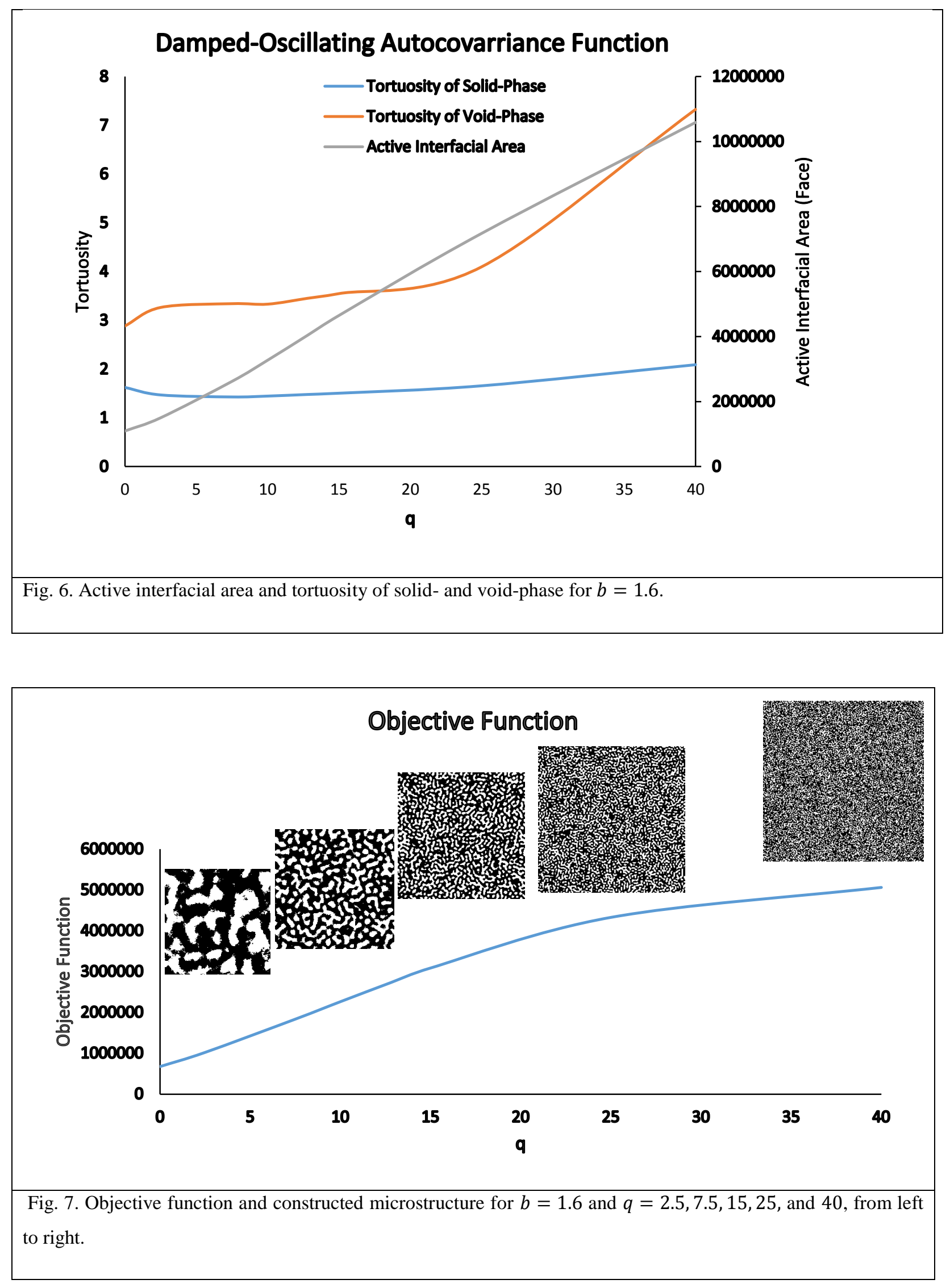
The trend of microstructure variation, while $b$ increases, is roughly similar to the Debye autocovariance function. The optimal design of the cathode microstructure based on the assumed objective function (Eq. (20)) for a specified void-phase volume fraction ( $v=0.41$ ) is achieved by compromising the interfacial area, tortuosity of solid-phase and constraint to the maximum void-phase tortuosity of 3.45 .

Microstructure with optimal active interfacial area has a high void-phase tortuosity and violate the maximum criteria of 3.45, and the one that has the lowest tortuosity has a low active interfacial area. By searching all domain of $0<b<51.2$ and $0<q<40$ and evaluating the objective function and satisfying the constraint of maximum void-phase tortuosity, the optimal design specification can be attained as in Table 1.

Table 1. Specifications of optimal design of cathode microstructure (O.F. stands for the objective function value).

\begin{tabular}{|l|c|c|c|c|c|c|c|}
\hline Microstructure type & $b$ & $q$ & $a$ (face) & $a_{\text {active }}($ face) & $\tau$ & $\tau_{\text {void }}$ & O.F. \\
\hline $\begin{array}{l}\text { Microstructure with optimal } \\
\text { tortuosity of solid-phase }\end{array}$ & 1.6 & 7.5 & 2673477 & 2618528 & 1.43 & 3.34 & 1835517.29 \\
\hline $\begin{array}{l}\text { Microstructure with optimal } \\
\text { objective function }\end{array}$ & 1.6 & 12.8 & 4065650 & 4038132 & 1.48 & 3.45 & 2732426.74 \\
\hline
\end{tabular}

3D illustration of optimal microstructures of Table 1 is depicted in Fig. 8. It should be mentioned that over 99\% of the interfacial area is active for these microstructures. Also almost all of the microstructures constructed using Eq. (9) have active interfacial ratio greater than 0.96. In addition, the number of active clusters for all constructed microstructure is one. It means that almost all solid- and void-phase sites are connected to each other.

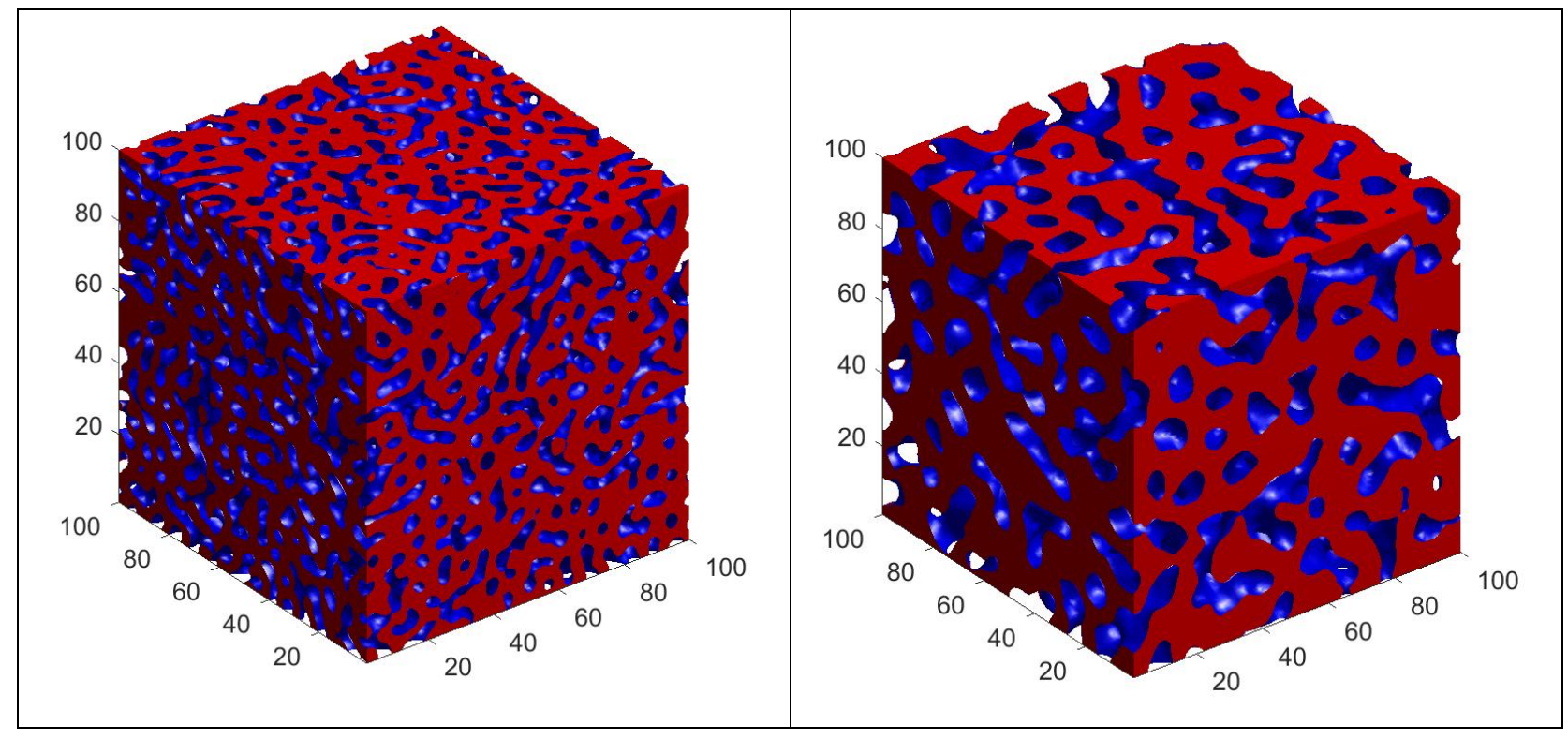


Fig. 8. Optimal design of cathode microstructure based on: left) objective function, right) solid-phase tortuosity (The void-phase is shown transparent).

For optimal microstructure, tortuosity of solid-phase compared to the work of Cooper et al. [42] gives a better result (about $17 \%$ for the same method, material and volume fraction). Also by equating the total RVE volume of the optimal microstructure equal to $70 \mu \mathrm{m}^{3}$, (similar to the work of Cooper [42]) the specific interfacial area of $12.15 \mu \mathrm{m}^{-1}$ can be obtained. This value is 2.4 times larger than that reported by Cooper et al. [42].

\section{Conclusions}

In the present study a systematic method was presented for the optimization of SOFC MIEC cathode microstructure based on the manipulation of the two-point correlation functions. Two-point correlation functions were used as a tool for providing various porous media and, therefore, a well-diversified search space. The autocovariance function that was used for calculating the TPCFs was a generalization of the Debye function that was consisted of an exponentially decaying function multiplied by an oscillatory (sine) function. Based on the feasibility of the autocovariance function, a two-variable search space was developed to represent various microstructures with diverse morphological and geometrical properties. Tortuosity of the solid-phase and the active interfacial area were selected as objective function in order to minimize the characteristic impedance of the cathode. Tortuosity as a factor that indicates the gas diffusion resistance of the void-phase was calculated based on the analogy between the heat transfer and diffusion equations. The optimization results revealed that by increasing the active interfacial area, the tortuosity, and therefore the gas diffusion resistance, was increased meanwhile the size of the void channels was decreased. Using the objective function, a microstructure with a reasonably optimized tortuosity and active interfacial area was obtained. In comparison with the experimental results reported in the literature, it was shown that the proposed procedure achieved an optimal microstructure with improved tortuosity in the solid-phase and larger active interfacial area.

\section{Acknowledgment}

The authors would like to acknowledge the funding for this Project from The Iran National Science Foundation (INSF).

\section{References}

[1] J. T.S. Irvine, P. Connor, Solid Oxide Fuels Cells: Facts and Figures, Springer-Verlag, London, 2013.

[2] J. Shi, X. Xue, Journal of Fuel Cell Science and Technology, 8 (2011) 061006-061006.

[3] X. Song, A.R. Diaz, A. Benard, J.D. Nicholas, Structural and Multidisciplinary Optimization, 47 (2013) 453-464.

[4] S. Farhad, F. Hamdullahpur, AIChE Journal, 58 (2012) 1248-1261.

[5] M.M. Sebdani, M. Baniassadi, J. Jamali, M. Ahadiparast, K. Abrinia, M. Safdari, International Journal of Hydrogen Energy, 40 (2015) 15585-15596. 
[6] M. Baniassadi, H. Garmestani, D.S. Li, S. Ahzi, M. Khaleel, X. Sun, Acta Materialia, 59 (2011) 30-43.

[7] J.S. Cronin, Y.-c.K. Chen-Wiegart, J. Wang, S.A. Barnett, Journal of Power Sources, 233 (2013) 174179.

[8] S.A. Tabei, A. Sheidaei, M. Baniassadi, F. Pourboghrat, H. Garmestani, Journal of Power Sources, 235 (2013) 74-80.

[9] H. Amani Hamedani, M. Baniassadi, A. Sheidaei, F. Pourboghrat, Y. Rémond, M. Khaleel, H. Garmestani, Fuel Cells, 14 (2014) 91-95.

[10] C. Sun, R. Hui, J. Roller, Journal of Solid State Electrochemistry, 14 (2010) 1125-1144.

[11] S. B. Adler, J.A.L. and, B.C.H. Steele, J. Electrochem. Soc., 143 (1996) 3554-3564.

[12] W. He, W. Lv, J.H. Dickerson, Gas Transport in Solid Oxide Fuel Cells, Springer, New York, 2014.

[13] M.T. Colomer, B.C.H. Steele, J.A. Kilner, Solid State Ionics, 147 (2002) 41-48.

[14] N. Ortiz-Vitoriano, C. Bernuy-López, I. Ruiz de Larramendi, R. Knibbe, K. Thydén, A. Hauch, P. Holtappels, T. Rojo, Applied Energy, 104 (2013) 984-991.

[15] P.M. Adler, C.G. Jacquin, J.A. QUIBLIER, Int. J. Multiphase Flow, 16 (1990) 691-712.

[16] A. Endo, S. Wada, C.J. Wen, H. Komiyama, K. Yamada, Journal of The Electrochemical Society, 145 (1998) L35-L37.

[17] Y. Jiao, F.H. Stillinger, S. Torquato, PHYSICAL REVIEW E, 77 (2008) 031135.

[18] K.K. Bodla, S.V. Garimella, J.Y. Murthy, International Journal of Heat and Mass Transfer, 73 (2014) 250-264.

[19] M.P. Bendsøe, N. Kikuchi, Computer Methods in Applied Mechanics and Engineering, 71 (1988) 197224.

[20] S. Torquato, S. Hyun, A. Donev, Journal of Applied Physics, 94 (2003) 5748-5755.

[21] S. Torquato, Optimal Design of Heterogeneous Materials, in: D.R. Clarke, M. Ruhle, F. Zok (Eds.) Annual Review of Materials Research, Vol 40, 2010, pp. 101-129.

[22] M.P. Bendsøe, O. Sigmund, Topology Optimization Theory, Methods, and Applications, 2 ed., Springer-Verlag Berlin Heidelberg, 2004.

[23] S. Torquato, Random heterogeneous materials: microstructure and macroscopic properties, Springer-Verlag, New York, 2002.

[24] B.L. Adams, S.R. Kalidindi, Fulwood, David T., Microstructure-Sensitive Design for Performance Optimization, Butterworth-Heinemann, Waltham, MA 02451, 2013.

[25] M.J. Beran, Statistical Continuum Theories, Monographs in Statistical Physics and Thermodynamics, Interscience, New York, 1968.

[26] M. Safdari, M. Baniassadi, H. Garmestani, S.A.-H. Marvan, JOURNAL OF APPLIED PHYSICS, 112 (2012) 114318.

[27] N. Sheehan, S. Torquato, Journal of Applied Physics, 89 (2001) 53-60.

[28] B.L. Adams, X. Gao, S.R. Kalidindi, Acta Materialia, 53 (2005) 3563-3577.

[29] S. Torquato, Journal of the Mechanics and Physics of Solids, 45 (1997) 1421-1448.

[30] A.M. Gokhale, A. Tewari, H. Garmestani, Scripta Materialia, 53 (2005) 989-993.

[31] S. Torquato, J. Chem. Phys., 111 (1999) 8832-8837.

[32] S. Torquato, (2006) 15-15.

[33] P. Debye, H.R. Anderson Jr, H. Brumberger, Journal of Applied Physics, 28 (1957) 679-683.

[34] D. Cule, S. Torquato, Journal of Applied Physics, 86 (1999) 3428-3428.

[35] Y. Jiao, F.H. Stillinger, S. Torquato, PHYSICAL REVIEW E, 76 (2007) 031110.

[36] A. Lanzini, P. Leone, P. Asinari, Journal of Power Sources, 194 (2009) 408-422.

[37] D.T. Fullwood, S.R. Kalidindi, S.R. Niezgoda, A. Fast, N. Hampson, Materials Science and Engineering A, 494 (2008) 68-72.

[38] D.T. Fullwood, S.R. Niezgoda, S.R. Kalidindi, Acta Materialia, 52 (2008) 942-948. 
[39] A. Hasanabadi, M. Baniassadi, K. Abrinia, M. Safdari, H. Garmestani, Computational Materials Science, 111 (2016) 107-115.

[40] R.W. Gerchberg, W.O. Saxton, OPTIK, 35 (1972) 237-246.

[41] J.R. Fienup, Optics Letters, 3 (1978) 27-29.

[42] S.J. Cooper, M. Kishimoto, F. Tariq, R.S. Bradley, A.J. Marquis, N.P. Brandon, J.A. Kilner, P.R. Shearing, ECS Transactions, 57 (2013) 2671-2678.

[43] J. Hoshen, R. Kopelman, Physical Review B, 14 (1976) 3438-3445. 
Creation the search space

Parameterized two-point correlation functions

$$
+
$$

Phase recovery algorithm

\section{Optimization}

Based on tortuosity of solid phase $\&$

Active interfacial area

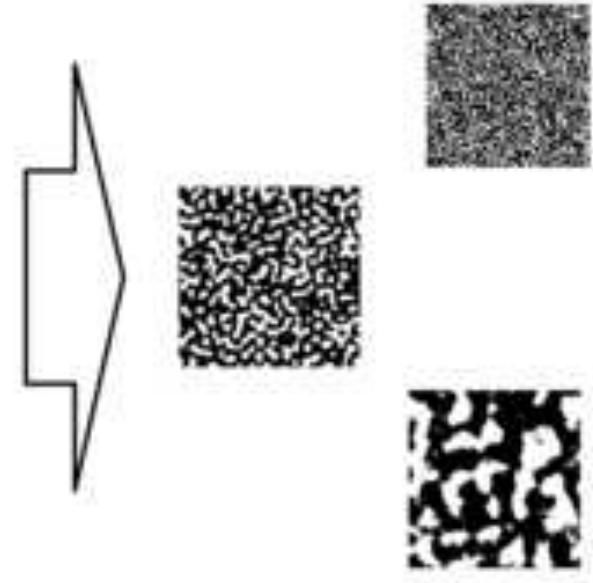

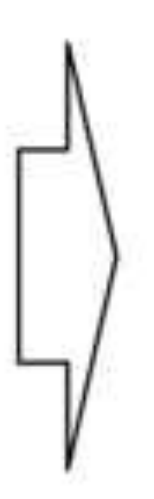

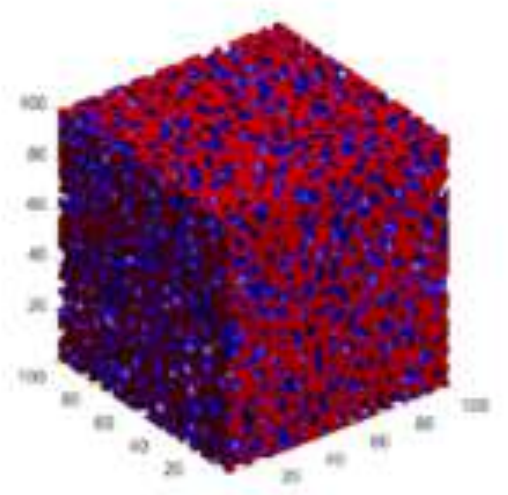

